\title{
Genetic and Demographic Consequences of a Rapid Reduction in Popula- tion Size in a Solitary Lemur (Mirza coquereli)
}

\author{
Matthias Markolf*,1, Christian Roos ${ }^{2}$ and Peter Kappeler ${ }^{1,3}$ \\ ${ }^{1}$ Institute of Zoology \& Anthropology, University of Göttingen, Berliner Strasse 28, 37073 Göttingen, Germany \\ ${ }^{2}$ Gene Bank of Primates and Department of Primate Genetics, German Primate Center, Kellnerweg 4, 37077 Göttingen, \\ Germany \\ ${ }^{3}$ Department of Behavioral Ecology \& Sociobiology, German Primate Center, Kellnerweg 4, 37077 Göttingen, Germany
}

\begin{abstract}
Population bottlenecks as a result of habitat fragmentation or other anthropogenic disturbances are increasingly common, but their consequences remain poorly studied. We studied the genetic population structure of a solitary nocturnal primate, Mirza coquereli, to investigate the demographic history of a local subpopulation in Kirindy Forest, western Madagascar, which showed recent dramatic fluctuations in population size. Additionally, we sampled the surrounding forest area to assess the genetic structure of the local population on a broader geographical scale. Analyses of mitochondrial DNA (mtDNA) and microsatellites of 53 individuals revealed that a recent reduction and subsequent recovery in population size (demographic bottleneck) in the local subpopulation did not result in a genetic bottleneck. Moreover, predicted matrilineal clusters and male-biased dispersal could be confirmed on a broader geographical scale by mtDNA analysis, but not by microsatellite analyses. These results suggest that a re-colonization of the demographic bottlenecked area took place. This study contributes to our knowledge of the genetic structure of solitary mammals and primates in particular and illuminates behavioral mechanisms, such as sex-specific dispersal, that influence the genetic structure of populations. Furthermore, we showed that populations of solitary mammals are able to compensate drastic local demographic changes via migration if habitat continuity at an appropriate scale is given.
\end{abstract}

Keywords: Bottleneck, population dynamics, mtDNA-microsatellites, conservation, re-colonization, migration, Mirza coquereli.

\section{INTRODUCTION}

The investigation of the genetic diversity of populations and the evolutionary forces that influence it is one of the most important topics for conservation management [1-3]. The number of individuals within a population determines its genetic diversity. A rapid reduction of population size, i.e. a demographic bottleneck, can result in loss of genetic diversity through genetic drift and inbreeding [4-6] if there is no compensation through gene flow [7]. In a natural environment gene flow between subpopulations is maintained by dispersing individuals and a small number of immigrants from neighboring populations can balance the loss of genetic variation in a bottlenecked population $[2,8]$. If migration potential decreases because of increased habitat fragmentation, inbreeding and eventually increased extinction risk could result [7]. Thus, information about the spatial genetic structure and resulting migration patterns that influence genetic diversity of populations is important for the planning and implementation of appropriate and effective conservation efforts. Most previous studies investigating genetic diversity and or dispersal patterns of mammalian species focused on the entire geographic range of a species or on small island populations. Gene flow in the form of dispersal, a

*Address correspondence to this author at the Department of Sociobiology/Anthropology, Institute of Zoology and Anthropology, University of Göttingen, Berliner Strasse 28, D-37073 Göttingen, Germany; Tel: ++49 551 397802; Fax: ++49 551 397299; E-mail: mmarkolf@web.de natural mechanism that could compensate for a loss of genetic diversity, cannot be considered at these scales. Therefore, measuring genetic population structure and diversity on hierarchical spatial scales, taking social organization into account, appears to represent a more meaningful approach $[9,10]$.

Until now, only a few studies have investigated the genetic consequences of a natural population bottleneck $[2,5$, 11], presumably because relevant genetic and demographic data from the past are often not available. Simulation studies violate key assumptions that have an influence on the genetic diversity of populations. For example, individuals do not die at random in a natural population and most natural populations are genetically not isolated as assumed by most simulated bottlenecks. We had the possibility to study the genetic consequences of a severe demographic bottleneck (note that we refer to a demographic bottleneck as a reduction of individuals $\mathrm{N}_{\text {census }}$ in a distinct spatial area and not as to a reduction in the effective population size $\mathrm{N}_{\mathrm{e}}$ ) in a natural population of a solitary primate, Coquerel's dwarf lemur (Mirza coquereli).

Mirza coquereli was given one of the highest priority rankings for conservation and represents also one of the least studied species among Malagasy lemurs [12]. Most of our knowledge about the biology and natural history of this species derives from a long-term study in Kirindy Forest, western Madagascar. Mirza coquereli is an approximately $300 \mathrm{~g}$ 
omnivorous nocturnal cheirogaleid that spends the day in self-constructed nests and forages solitarily at night [13-15]. Adult male and female Mirza inhabit individual home ranges of about 1-4 ha in size, which overlap with home ranges of up to eight neighbors [16]. Male home ranges in Kirindy were observed to more than quadruple in size during the mating season. Mating in Kirindy is restricted to a few weeks in October - November and females typically give birth to 2 young after a gestation period of approximately 90 days $[17,18]$. Mixed paternities, large testes and the fact that males roam widely beyond their core ranges during the mating season indicate that the mating system of Mirza is best described as scramble competition polygyny [16].

The local population density of Mirza decreased dramatically over the time course of our long-term study in Kirindy (see Appendix A in [14]). During the years 1999-2003, only two animals could be trapped in a study area that had housed up to 30 individuals before. Afterwards, the population recovered to its previous density, suggesting that this population possibly also underwent a genetic bottleneck.

Kappeler et al. [14] already used genetic methods to assess the socio-genetic structure of this local Mirza population. They found a matrilineal structure in this solitary primate. Females sharing a mtDNA haplotype were clustered in space and relatedness was negatively correlated with geographical distance in females, but not in males. These results indicated female philopatry, but facultative female dispersal was observed, as well. For Mirza males, philopatry and dispersal also appeared to be facultative options. For example, one male was born within the study area and remained there for at least three years. Three other males with unique haplotypes were also found in the subpopulation under study [14], indicating that male dispersal over large areas is possible and realistic. However, only one of these males stayed there for more than one year. Kappeler et al. [14] argued that males could have problems in establishing themselves outside their natal range, or that secondary dispersal is common. However, patterns of dispersal needed also further investigation, especially with respect to the demographic changes of the local subpopulation.

The aim was to study the population structure of Mirza coquereli at different spatial scales in order to investigate: (1) the demographic history of the local subpopulation that underwent a demographic bottleneck, (2) the genetic consequences of the demographic bottleneck and (3) the sexspecific genetic structure on a broader geographical scale as it relates to dispersal patterns.

\section{MATERIALS AND METHODS}

\section{Field Work and Sample Collection}

Tissue samples were collected in Kirindy Forest, a dry deciduous forest, approximately $60 \mathrm{~km}$ northeast of Morondava in western Madagascar, where the German Primate Center has operated a field station since 1993. The field station includes three grid systems with small foot trails every 25(50) $\mathrm{m}$ named N5, CS5 and CS7, each of them at least 25 ha in size (Fig. 3). Since 1993, Mirza have been regularly trapped within the grids N5 and CS7 with Sherman life traps (for details see [16]). In 2005/2006 individuals were additionally trapped on several transects between the grid sys- tems, in CS5 and outside Kirindy Forest, up to $30 \mathrm{~km}$ away from the other sites. The distance between traps on transects of $50 \mathrm{~m}$ was defined by a Garmin etrex GPS, which had an accuracy of 4 to $9 \mathrm{~m}$. Every newly trapped animal was anesthetized with $0.04 \mathrm{ml}$ Ketanest, individually marked with a transponder (Trovan Small Animal Marking System; Telinject, Römerberg, Germany) and subjected to standard morphometric field measurements. Additionally, small ear biopsies $(2-3 \mathrm{~mm})$ were taken during anesthesia and the tissue samples were immediately stored in $70 \%$ ethanol for later DNA analyses.

\section{Genetic Analyses}

Tissue samples from 69 individuals were available for this study. Three animals were trapped in 2001, eleven in 2004 and all others in 2005/2006. 34 samples were available from the population in N5 that underwent a demographic bottleneck. Only one animal was trapped in 2000 and 2001 in N5 and again in 2005 and 2006. Unfortunately no DNA of this individual was available. Another individual was trapped in 2001 and 2004/2005. Since this individual was included in the analysis, data from 2001- 2006 were grouped. Fig. (1) shows the number of individuals trapped in the main study area from 1993-2006. All data from the pre-bottlenecked population are from Kappeler et al. 2002. Genomic DNA was extracted using the QIAamp Tissue Kit from QIAGEN following instructions of the company.

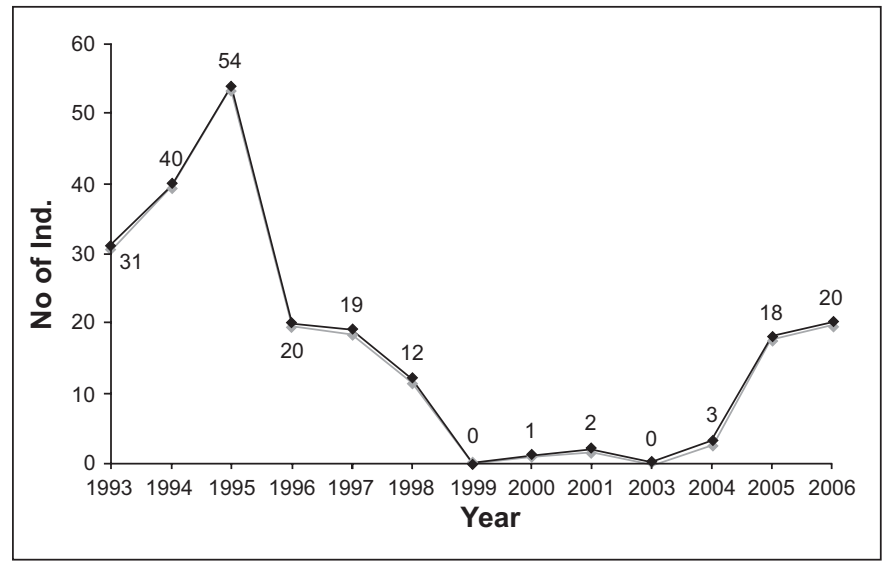

Fig. (1). Number of Individuals trapped in the Grid N5 from 1993- 2006.

Note that the total number of individuals from 2001- 2006 does not equal the sample size of 34 used for genetic analyses, because some Individuals were trapped in more than one year or had to be excluded from analyses.

\section{Mitochondrial DNA}

A 720bp long fragment of the hypervariable region I (HVI) of the mitochondrial D-loop was amplified with oligonucleotide primers as described [19]. In order to exclude miss-amplification of nuclear pseudogenes ("numts"), an alternative primer pair [14] was used to amplify a shorter fragment (ca. 530bp) of the (HVI) region.

PCR reactions were performed in a total volume of $30 \mu \mathrm{l}$ and consisted of $14,3 \mu 1 \mathrm{H}_{2} \mathrm{O}, 0,5 \mu \mathrm{l}$ DNA Taq polymerase (Biozym), $1 \mu \mathrm{l}$ of each primer (10pmol/ $\mu \mathrm{l}), 0,2 \mu 1 \mathrm{dNTP}$ 's

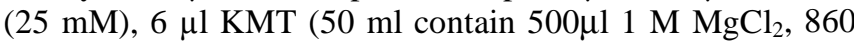


$\mu 11 \mathrm{M} \mathrm{HCL}, 5 \mathrm{ml} 2,5 \mathrm{M} \mathrm{KCL}, 2,5 \mathrm{ml} 1 \mathrm{M}$ Tris and $\left.\mathrm{H}_{2} \mathrm{O}\right), 4$ $\mu \mathrm{l}$ BT $(50 \mathrm{ml}$ contain $5 \mathrm{ml}$ BSA $100 \mathrm{mg} / \mathrm{ml}, 250 \mathrm{mg}$ Triton $\mathrm{X} 100$ and $\left.\mathrm{H}_{2} \mathrm{O}\right)$ and $2 \mu \mathrm{l}(25-75 \mathrm{ng})$ DNA. Cycling conditions were as follows: initial denaturation step of $2 \mathrm{~min}$ at $94^{\circ} \mathrm{C}$, followed by 35 cycles each with $30 \mathrm{~s}$ at $94^{\circ} \mathrm{C}, 45 \mathrm{~s}$ at $56^{\circ} \mathrm{C}, 60 \mathrm{~s}$ at $72^{\circ} \mathrm{C}$. At the end, a final extension step of 5 min at $72^{\circ} \mathrm{C}$ was added. PCR products were purified with the Millipore DNA Purification Kit and sequenced on an automated capillary sequencer (ABI3100-Avant, Applied Biosystems) using the BigDye Terminator Cycle Sequencing Kit and the primers as mentioned above. Sequences were aligned and edited using SEQUENCHER 4.7 and trimmed to 529bp to allow comparison with haplotypes of Kappeler et al. [14]. Identical haplotypes were found using COLLAPSE version 1.2. [20].

\section{Microsatellites}

Microsatellites were established via cross-species amplification using primer pairs originally derived from Microcebus murinus (Mm22, Mm42, Mm53 [21], Mm58 [22], C1P3, Mm08 [23]) and from Cheirogaleus medius (110 [22]). In a $20 \mu \mathrm{l}$ reaction, 14,34 $\mu \mathrm{l} \mathrm{H}_{2} \mathrm{O}, 2 \mu \mathrm{l} 10 \mathrm{x}$ buffer (contains 15 $\mathrm{mM} \mathrm{MgCl}_{2}$, Biotherm) $0,2 \mu \mathrm{l}$ of each primer (100pmol $\left./ \mu \mathrm{l}\right)$, $0,16 \mu 1$ dNTP's $(25 \mathrm{mM}), 0,1 \mu \mathrm{l}$ DNA polymerase (Biotherm, $5 \mathrm{u} / \mu \mathrm{l})$ and $3 \mu \mathrm{l}$ (50-100ng) DNA was used. PCR programs followed those as described. After 1:10 or 1:5 dilution, 0,7 $\mu 1$ of each PCR product was analyzed on an ABI 377 DNA sequencer along with a labeled standard (Genescan 400 HD [Rox] Size Standard). Genotypes were scored with the programs ABI Prism 377-96 Collect and GeneScan version 3.1.2.

\section{Spatial Analyses}

Using ArcView version 3.1, we created haplotype distribution maps of all captured animals. The mean coordinates of all capture sites for a given individual were used for spatial analyses.

\section{Statistical Analyses}

\section{General Analyses}

For all population genetic analyses only the 53 individuals trapped within the three grid systems and on nearby transects (see Fig. 3) were used. All other Mirza captured further away provided additional information for haplotype distribution, but we have no information on Mirza abundance at these sites. Because the three grid systems are regularly trapped and there have been several trapping sessions along transects by other researchers in the last years (e.g. see [9]), one can argue that the samples obtained in this study reflect a realistic image of the distribution of individuals of Mirza coquereli within this area.

Using DnaSP 4.10.3 [24] we calculated standard population genetic parameters for haplotypes, such as haplotype diversity and nucleotide diversity. Tests for Hardy Weinberg Equilibrium (HWE) were performed using the Chi-square test implemented in GenAlEx [25] and test for linkage disequilibrium was conducted using FSTAT version 2.9.3.2 [26]. MICRO-CHECKER [27] was used to test for the presence of null alleles. MICRO-CHECKER also provides an opportunity to adjust genotypes and allele frequencies of loci with null alleles present.

\section{Demographic History}

To investigate whether the population in N5 has undergone a genetic bottleneck, we calculated several diversity indices of the mitochondrial D-loop, using DnaSP for both, the datasets from 1993-1998 [14] and 2001-2006. Additionally, two summary statistics that measure the neutrality of sequence evolution by comparing different estimators of genetic diversity were calculated. These statistics are also influenced by demographic changes of populations. Tajima's D compares the number of segregating sites (Theta) and the average nucleotide diversity (Pi) [28]. Fu and Li's D* [29] compares the number of singletons (mutations that appear only once among the sequences) and the total number of mutations. These statistics tend to be zero if a population is panmictic and stable in size, and deviate from zero if sequence evolution is under selection or demographic fluctuations occurred. Negative values reflect an excess of rare polymorphisms, which could be explained by positive selection or by the fact that the population has undergone a drastic change in population size (genetic bottleneck). Positive values reflect an excess of alleles with intermediate frequencies and could be explained by balancing selection or population fusion. DnaSP was also used to conduct a mismatch distribution. Here the distribution of pairwise nucleotide site differences is plotted and these distributions are characteristic for population with different demographic histories. A bimodal shape (genetic diversity is only reduced) and a L-formed shape (genetic diversity is completely removed) indicate that the population has undergone a bottleneck [7]. Populations characterized by exponential growth, such as humans, have a unimodal shape and stable populations a multimodal and ragged shape [30].

A test for a genetic bottleneck was conducted with the microsatellite data set, using the program BOTTLENECK [31]. Populations that have experienced a recent reduction in their effective population size show a correlative reduction of allele numbers and heterozygosity at polymorphic loci, but the allele number is reduced faster than heterozygosity. By simulating a coalescent process of $n$ genes, the distribution of the expected heterozygosity (calculated from allele numbers and sample size) for each sample and each locus is calculated and can be compared with the HW heterozygosity (calculated from allele frequencies). Bottlenecked populations should show heterozygosity excess at each locus. Because we had only 7 polymorphic loci, the recommended Wilcoxon's test [31] gives the most reliable results. Because it was obvious from visual inspection that the microsatellite loci do not fit a strict stepwise mutation model, the recommended two-phase-model (TPM), with 95\% single step mutations and 5\% multi-step mutations and variance among multiple steps of 12 was chosen. BOTTLENECK also provides a graphical output of the shape of the allele frequency distribution, which differentiates between bottlenecked and stable populations. Bottlenecked populations show a modeshift to more alleles at intermediate frequencies, whereas stable populations are characterized by a L-shaped distribution, thus, showing more alleles with low frequencies [32]. The analysis was repeated with adjusted allele frequencies for Mm08 and 110, because they were considered to might have null alleles present. 


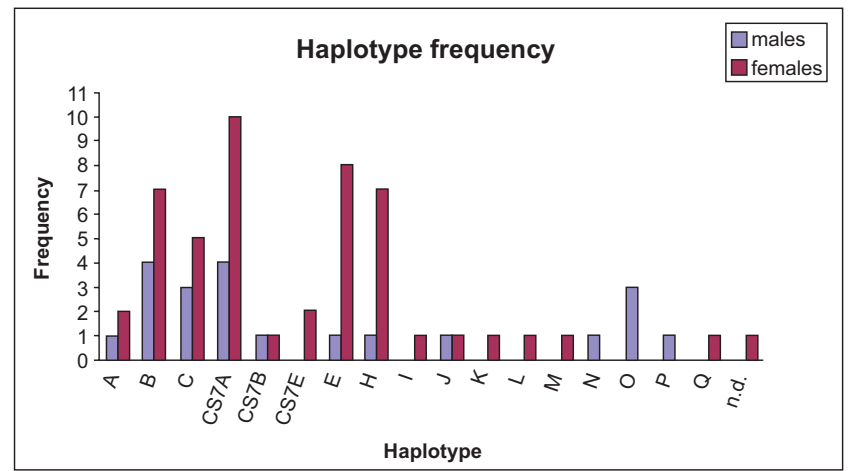

Fig. (2). Haplotype frequencies of all males and females trapped since 2001.

Alphabetic characters represent different haplotypes, males=blue, females $=$ red, .. . $=$ not determined.

\section{Spatial-Genetic Structure}

To investigate the sex-specific population structure, we performed spatial genetic autocorrelations for adult males (8) and females (17) separately over the total population with both markers, using also GenAlEx. First a genetic and geographic distance matrix was calculated $[33,34]$ which was then used to calculate an autocorrelation coefficient $\mathrm{r}$ ( -1 to +1 ). $\mathrm{r}$ provides a measure of genetic similarity between pairs of individuals belonging to the same distance class. The program also calculates by permutations the $95 \%$ upper and lower confidence intervals and the standard deviation for each $\mathrm{r}$-value by bootstrapping $(\mathrm{N}=999)$. This results in $\mathrm{r}$ values for each a priori defined distance class, which become connected to a curve in the correlogram output. rvalues higher than the upper $95 \%$ confidence interval indicate positive spatial structure, i.e., individuals are more genetically similar as expected for a random distribution. We choose distance classes of $200 \mathrm{~m}$, which represent a biological meaningful distance class for a species with overlapping home ranges of 1-4 ha in size. The analyses were repeated without loci C1P3, Mm08 and 110, because those loci were considered to might have null alleles present.

\section{RESULTS}

\section{General Results}

Mitochondrial sequences derived from the two different PCR products were identical, indicating that missamplification of numts can be excluded. Among the 69 genotyped individuals, we identified 17 different haplotypes. This resulted in a haplotype diversity $(\mathrm{Hd})$ of 0.895 . Nucleotide diversity (Pi) was 0.017. Haplotype H, I, J, K, L, M, N, O, P and Q were new haplotypes (Fig. 2), but only H, I, K and Q were found within the grids. Individuals with haplotypes A and $\mathrm{C}$ were also trapped approximately $8 \mathrm{~km}$ from the grid area and haplotypes B, CS7A and CS7E were found even more than $30 \mathrm{~km}$ away from the main study site. Fig. $(\mathbf{3 a}+\mathbf{b})$ show distribution maps of haplotypes that were used for population genetic analyses. Haplotypes are available at Genbank (Accession numbers: EU518379- EU518398).

The seven microsatellite loci were highly polymorphic and showed, except for locus Mm42, significant deviations from HWE and heterozygosity deficiency. Summary statistics for each locus are listed in Table 1. The test for linkage equilibrium revealed independent segregation of each locus. MICRO-CHECKER revealed that loci C1P3, Mm08 and 110 were likely to have null alleles present. For N5 only Mm08 and 110 were considered as those.

\section{Demographic History}

The comparison of haplotypes of the N5 population before and after the reduction in population size showed a frequency shift to other haplotypes (Fig. 4). Haplotype E was formerly only represented by one male and is now highly represented in the population. Haplotype A was formerly highly represented and is now only represented by three individuals outside of N5. Furthermore, haplotypes D, F, G are not represented anymore and the new haplotypes $\mathrm{H}$ and CS7A are very common. a

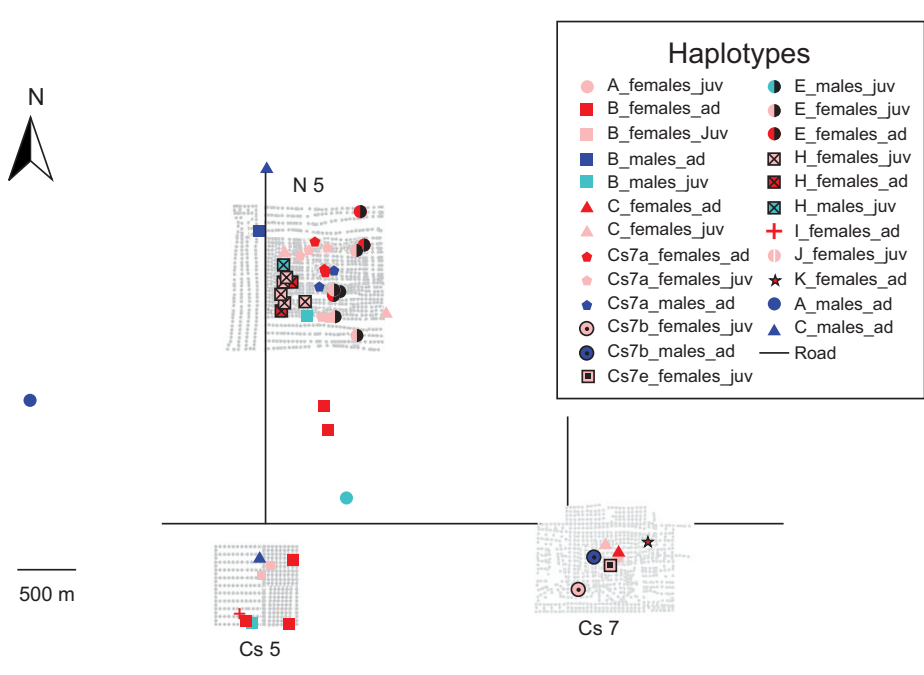

b

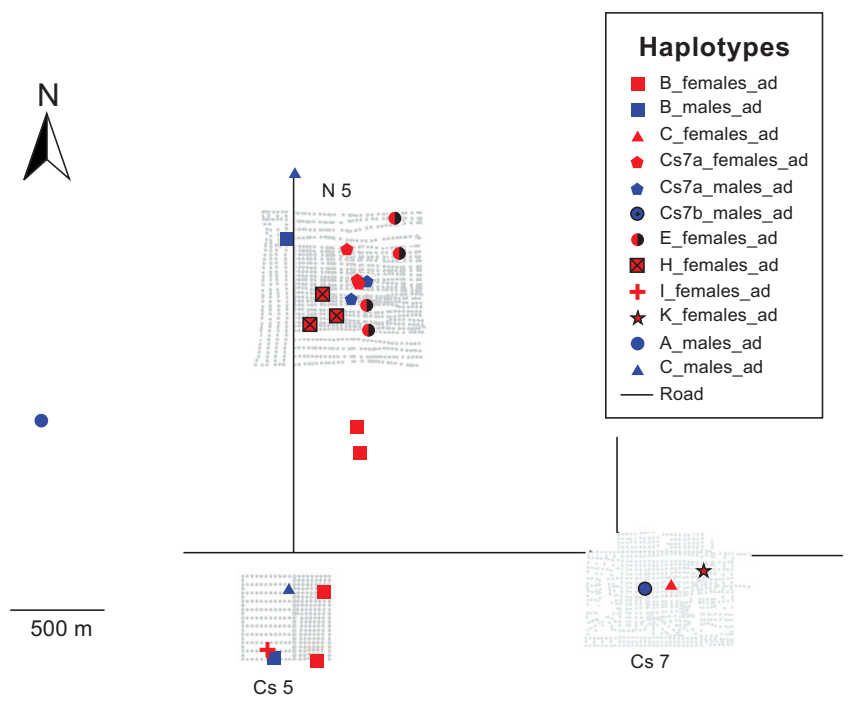

Fig. (3). a+b Haplotype distribution of captured animals used for population genetic analyses.

a) adults and juveniles, $\mathbf{b}$ ) only adults, males $=$ blue, females $=$ red, symbols $=$ haplotypes . 
Table 1. Summary Statistics for Microsatellite Loci and HWE Test

\begin{tabular}{|c|c|c|c|c|c|c|c|c|}
\hline \multicolumn{1}{|c|}{ Locus } & N & Na & Ho & He & Fis & DF & ChiSq & Sign. \\
\hline \hline C1P3 & 53 & 23 & 0,792 & 0,894 & 0,113 & 253 & 610,580 & $* * *$ \\
\hline Mm08 & 53 & 20 & 0,698 & 0,892 & 0,217 & 190 & 371,849 & $* * *$ \\
\hline Mm58 & 53 & 14 & 0,774 & 0,847 & 0,087 & 91 & 155,711 & $* * *$ \\
\hline $\mathbf{1 1 0}$ & 53 & 10 & 0,415 & 0,649 & 0,360 & 45 & 180,905 & $* * *$ \\
\hline Mm22 & 53 & 15 & 0,717 & 0,741 & 0,032 & 105 & 194,800 & $* * *$ \\
\hline Mm53 & 53 & 14 & 0,736 & 0,795 & 0,075 & 91 & 229,011 & $* * *$ \\
\hline Mm42 & 53 & 9 & 0,849 & 0,777 & $-0,093$ & 36 & 26,135 & $\mathrm{~ns}$ \\
\hline
\end{tabular}

$\mathrm{ns}=$ not significant, $* * * \mathrm{P}<0.001, \mathrm{~N}=$ Number of individuals, $\mathrm{Na}=$ Number of alleles, $\mathrm{Ho}=$ Heterozygosity observed, He $=$ Heterozygosity expected, Fis $=$ inbreeding coefficient, DF= Degrees of freedom, $\mathrm{ChiSq}=\mathrm{Chisquared}$ value.

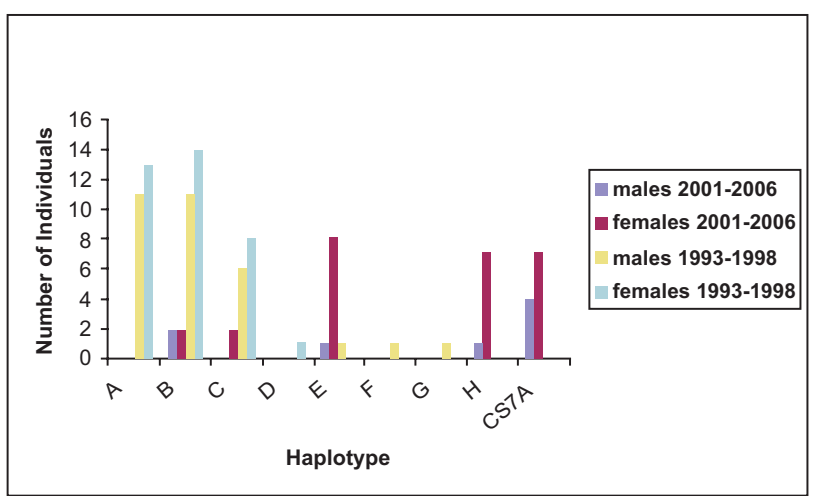

Fig. (4). Comparison of the number of haplotypes before and after the reduction of population size in $\mathbf{N 5}$. Note the shift from formerly highly represented haplotypes to new or formerly underrepresented haplotypes. Haplotypes from 1993-1998 are from Kappeler et al. 2002 [14].

The comparison of the genetic diversity indices did not reveal a reduction of genetic diversity (Table 2 ). Only the number of polymorphic sites and the number of singletons was clearly reduced. Tajima's D and Fu and Li's D* were both positive after the demographic bottleneck. Fu and Li's D* was significant, which is consistent with either balancing selection or population fusion. The mismatch distribution revealed a multimodal and ragged shape, assumed for demographic equilibrium or a stable population (data not shown).

The microsatellite analyses for demographic history of the N5 population revealed consistent results with the mtDNA analyses. The Wilcoxon's one-tailed test for heterozygosity excess was not significant (Wilcoxon testStatistic $=\mathrm{p}=0.996)$. Moreover, the mode-shift distribution showed a normal L-shaped distribution, as is expected for stable populations (data not shown). A rerun, with adjusted allele frequencies for loci Mm08 and 110 revealed congruent results (data not shown).

\section{Spatial Genetic Structure Dispersal}

The spatial genetic autocorrelation analyses with the mtDNA (Fig. 5a+b) revealed a significant positive spatial structure for adult females within a geographic distance of approximately $300 \mathrm{~m}$, indicating that proximate females share a mitochondrial haplotype. In contrast, for adult males no deviation from a random distribution was detectable. However, the same analyses for adult males and females using the microsatellite dataset failed to detect any deviation from a random distribution, neither for adult males, nor for adult females (Fig. $\mathbf{5 c + d}$ ).

Table 2. Comparison of Diversity Indices

\begin{tabular}{|l|c|c|}
\hline Diversity indices N5 & 1993- 1998* & 2001- 2006 \\
\hline \hline Number of sequences/individuals & 67 & 34 \\
\hline Number of haplotypes (in common) & 7 & $5(3)$ \\
\hline Polymorphic sites & 29 & 19 \\
\hline Haplotype diversity (Hd) & 0.698 & 0.763 \\
\hline Nucleotide diversity (Pi) & 0.014 & 0.013 \\
\hline Singletons & 11 & 1.657 \\
\hline Tajima's D & 0.705 & $\mathrm{p}>0.1$ \\
\hline Sign. & $\mathrm{p}>0.1$ & 1.644 \\
\hline Fu and Li's D* & -1.466 & $\mathrm{p}<0.02$ \\
\hline Significance & $\mathrm{p}>0.1$ & 02 \\
\hline
\end{tabular}

Shown are the diversity indices of the N5 population before and after the reduction in population size. Statistical significance of the D-values was obtained according to Tajima [28] and Fu and Li [29]. * Sequences from Kappeler et al. [14].

From visual inspection it is obvious that haplotypes are homogeneously distributed. Four of them are found (A, B, C, CS7A) in at least two of the grids. This and the fact that no spatial genetic structure could be detected with the microsatellite dataset indicate that migration among the grids is strong. A repetition of the analyses without those microsatellite loci, which might have null alleles present, did not change the results (data not shown).

\section{DISCUSSION}

The most important results of this study are that (1) the subpopulation in N5 still exhibits high levels of genetic diversity, so that a recent genetic bottleneck following a 


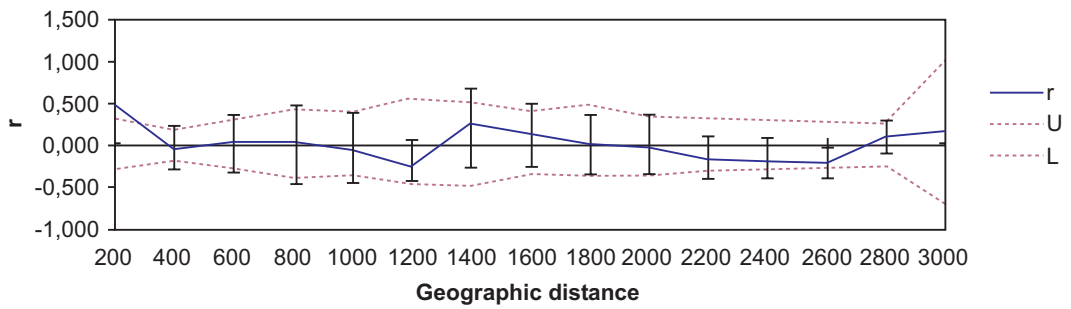

b

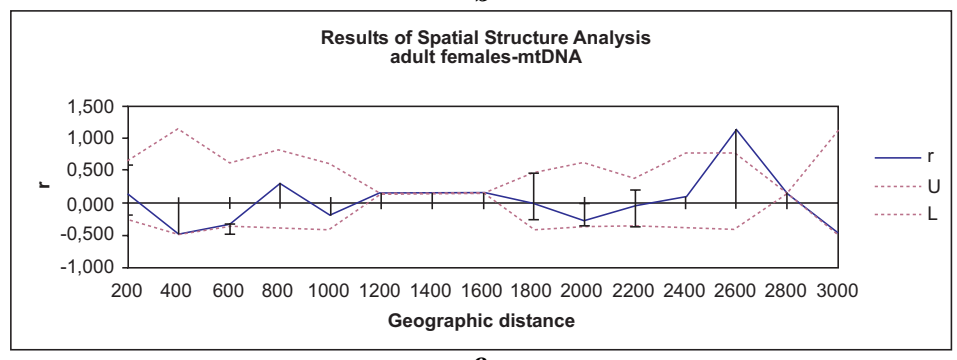

Results of Spatial Structure Analysis adult females-microsatellites
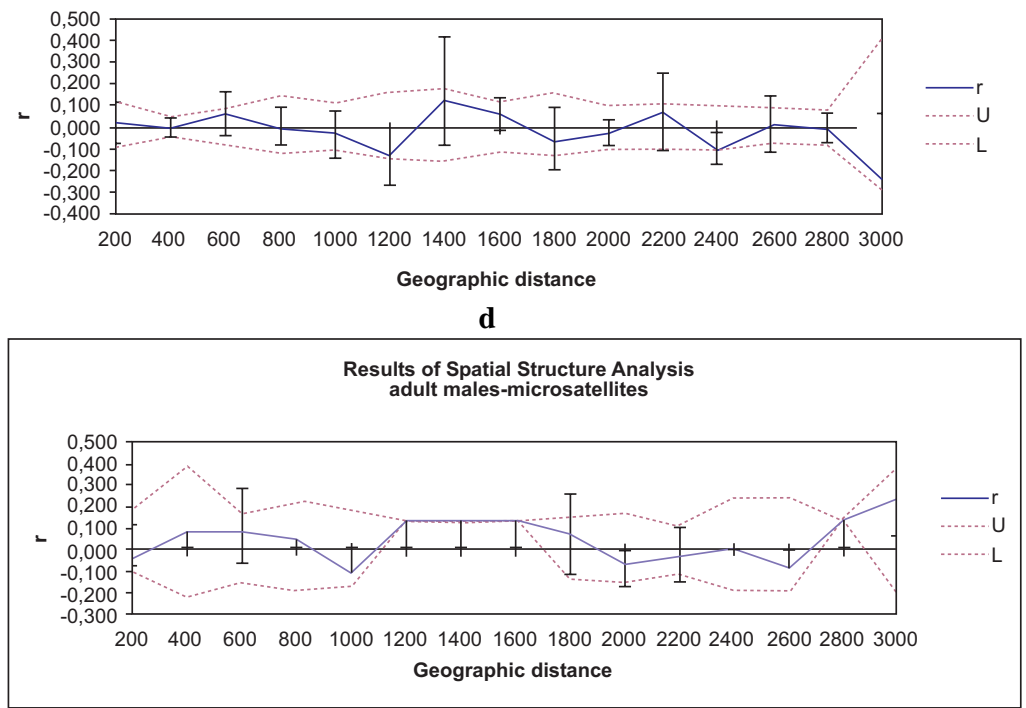

Fig. (5). a-d Spatial genetic autocorrelation correlograms.

The autocorrelation coefficient $r$ is plotted against the spatial distance class (geographic distance in $\mathrm{m}$ ). a) adult females-mtDNA b) adult males-mtDNA, c) adult females-microsatellites, d) adult males-microsatellites. The dotted red lines represent the $95 \%$ confidence intervals ( $\mathrm{U}=$ Upper, $\mathrm{L}=$ Lower) for a random distribution. The standard deviation obtained by bootstrapping is also depicted for each $\mathrm{r}$ (autocorrelation coefficient=blue line) value. Note that there is significant positive spatial structure in adult females for mtDNA but not for microsatellites.

demographic bottleneck can be excluded, (2) an organization into matrilineal clusters was also evident on a broader geographical scale than previously reported [14], and (3) there is high migration between subpopulations about $2 \mathrm{~km}$ apart from each other.

\section{Demographic History}

All tests for a genetic bottleneck with mtDNA and microsatellites revealed congruent results, namely that a genetic bottleneck is not evident in the local subpopulation of N5. The most plausible explanation for this lack of a bottleneck is that the area was re-colonized by individuals from the sur- rounding area. Best evidence for this re-colonization comes from the fact that the haplotype distribution showed a modeshift to formerly underrepresented haplotypes, which also underlines the potential of genetic drift in bottlenecked populations, and that there are at least two new haplotypes in the area. One of them was formerly found in the grid of CS7 about $2 \mathrm{~km}$ away. We know of at least one female that was trapped as a juvenile in 1998 and was still living in the area in 2006. Unfortunately, DNA amplification failed for this individual. Nevertheless, mother- daughter interactions (pers. observation) indicate that this female had haplotype CS7A, the most abundant haplotype after the bottleneck. Because 
this is the only individual that outlived the time of absence of other individuals, it is unlikely that the entire population emigrated to the surrounding area and then returned.

It remains obscure what could have caused the reduction in population size. Because trapping in this area has been conducted regularly since 1993, one can exclude the possibility that the reduction in population size is simply an artifact of reduced trapping effort. Instead, individuals may have left the area because of variation in ecological factors, such as food availability, but this explanation does not accord with data on other sympatric cheirogaleids, which were highly abundant throughout the same time and which use similar resources [9, 35-37]. Mirza is also exposed to high predation pressure (personal observation). Predator-prey interactions can result in drastic fluctuations of population density, but this is also unlikely if one takes sympatric species into account, which are also exposed to high predation pressure from the same predators, but did not show these fluctuations. Thus, the question cannot be answered with the data at hand because specific ecological data, for example on food abundance or pathogen abundance, are missing.

\section{Spatial Structure}

The spatial genetic autocorrelation analysis for the mtDNA could confirm the matrilineal structure already detected by Kappeler et al. [14] on a broader geographical scale. Females with identical haplotypes were significantly clustered in space within a spatial distance class of approximately $300 \mathrm{~m}$. Although standard deviations are large for each size class, and the r-line barely exceeds the upper confidence limit at $200 \mathrm{~m}(\mathrm{r}=0,501 \mathrm{U}=0,34)$ the positive spatial structure is supported by the fact that even the lower standard deviations remain positive. The analysis of microsatellites failed to confirm this pattern, however. The same pattern re-appeared, however, when we performed Mantel's tests for adult males and females separately (data not shown). Discrepancies between mtDNA and nuclear genomes can arise if gene flow is sex-biased. Because mtDNA is only maternally inherited, higher dispersal of males compared to females results in a more homogeneous distribution of nuclear DNA, compared to mtDNA. In mammals, for example Escorza-Trevino and Dizon [38] for porpoises, Nyakaana and Arctander [39] for elephants and Muwanika et al. [40] for warthogs, also found lower levels for subdivision of populations for microsatellites than for mtDNA, which was associated with male-biased dispersal (see also Sanetra and Crozier [41] for ants or Haavie et al. [42] for the pied flycatcher).

We also performed an Analysis of Molecular Variance (AMOVA) using GenAlEx with both markers splitting the total populations in three subpopulations (grids) and two regions (N5, CS5 and intercostals individuals vs. CS7). Both markers showed congruently most variation within the subpopulations (mtDNA $88 \%$, microsatellites 95\%), indicating that there is a high amount of gene flow within the total area. However, due to the small sample size, results are not significant (data not shown).

Because two haplotypes in the N5 population were new to the area, they could only have been introduced by female migration. We do not know anything about the detailed dispersal distance of these females, demonstrating the impor- tance of combining molecular genetic studies with behavioral observations to identify dispersal patterns of individuals [43]. Finally, dispersal in M. coquereli was again found to be male-biased, but this is not a strict rule without exceptions.

\section{Deviations from HWE, Null Alleles and Sample Size}

It needs to be mentioned that six out of the seven loci were found not to be in Hardy-Weinberg proportions and showed heterozygosity deficits over the total population. Deviations from HWE can arise if selection or assortative mating is present or if subpopulations are treated as one population without reproduction (Wahlund effect). A Wahlund effect can be excluded because high migration was evident from the distribution of haplotypes, therefore reproductive isolation between "subpopulations" is unlikely. Increased homozygosity due to mating among relatives is also unlikely because no spatial-genetic structure was found with microsatellites. Additionally, deviations from HWE can arise if null alleles are present and some heterozygotes become homozygotes. MICRO-CHECKER revealed that C1P3, Mm08 and 110 showed homozygosity excess over the total population, so that null alleles may be present. MICROCHECKER also provides an opportunity to adjust the genotypes and allele frequencies. Genotype adjustment is done at random over homozygotes of the population, making spatial analyses with adjusted genotypes useless. Thus, we repeated microsatellite analyses without these markers and obtained similar results. For the demographic analysis it was also possible to use the adjusted allele frequencies of the N5 population. For N5 only Mm08 and 110 were considered to have null alleles. The analysis revealed congruent results with the other analysis. There was no evidence for a genetic bottleneck.

Finally, the overall sample size for population genetic analyses of 53 individuals was not very large and we still know little about the distribution of individuals in the surrounding area. At the moment the most plausible explanation for deviations from HWE is that our samples represent only a small fraction of the true population. Small sample size also raises the question whether conclusions of population genetic analyses are robust. For example, it could be argued that with such a small sample size a mismatch distribution is likely to be multimodal [see 44]. Another example is the positive spatial structure of male mtDNA in the spatial genetic autocorrelation at $2600 \mathrm{~m}$. Accidentally, these $2 \times 2$ males share the same haplotype and those individuals are the only data points for this size class. Accordingly, the test shows positive spatial structure, which can be considered as random by looking at the haplotype distribution map.

Moreover, the analyzed seven microsatellite markers might not be sufficient for population genetic analyses. Overall, we tested 17 different primer pairs of closely related lemur species (Microcebus murinus, Cheirogaleus medius), but only seven of those were amplififiable and polymorphic in Mirza coquereli. Although generating species-specific microsatellite primers is possible, the quality and quantity of our DNA was not sufficient for this purpose. Indeed, few markers and small sample size are likely to produce errors in population genetic studies, thus we did not rely on just one analysis and genetic markers from two different genomes were studied. All analyses revealed congruent results, so that 
a genetic bottleneck is very unlikely. Moreover, in case of the spatial genetic autocorrelation, small sample size is unlikely to lead to wrong results, because we confirmed the matrilineal structure already shown by Kappeler et al. [14] on another geographical scale.

\section{Conservation Aspects}

This study was conducted on a larger spatial scale than the study of Kappeler et al. [14] and revealed that M. coquereli, like sympatric $M$. murinus $[9,36]$, is also distributed in patches, indicating that a continuous forest does not always provide a continuous suitable habitat. In the gray mouse lemur, Microcebus murinus, closely related females sleep together during the day and members of sleeping groups share a mitochondrial haplotype. The outcome is again a pattern of spatially distributed matrilineal clusters. Most adult males within such matrilines have a different mtDNA haplotype [45, 46]. Fredsted et al. [9] demonstrated that such clusters or population nuclei of closely related individuals were also found on a broader geographical scale and that gene flow between these nuclei is primarily caused by male-biased dispersal. Furthermore, they found that the population is structured into different breeding groups. This pattern was not evident in previous studies on smaller geographical scales and this is also not the case in Mirza coquereli.

In contrast, the distribution of haplotypes in this study was more homogeneous, indicating that gene flow between patches that are approximately two kilometers apart occurs. A more homogeneous distribution is also indicated by the absence of spatial structure in the microsatellite dataset. The results indicate that $M$. coquereli is able to compensate local demographic fluctuations via high migration. Unlike isolated fragments, continuous forests that harbor multiple population nuclei allow gene flow via migration of individuals, thereby preventing inbreeding depression and loss of genetic diversity [7]. Thus, in relatively undisturbed continuous habitat, a solitary species is able to help itself in maintaining genetic diversity. The genetic variability that was found on the broader geographical scale in this study was not evident from the previous study on a smaller local subpopulation, which underlines the importance of studying genetic structure of populations on different spatial scales and not only in a randomly chosen small population.

This study covered an area of approximately 300ha. The fact that the density of Mirza is much lower than that of other smaller sympatric cheirogaleids and that Mirza is vulnerable to drastic demographic fluctuations, we argue that a Mirza population needs at least a three to fourfold area of continuous forest to maintain its genetic diversity. The distribution of Mirza coquereli is restricted to the western dry deciduous forests of Madagascar, one of the most rapidly declining forest areas of the world [47]. The fact that only a few forest fragments of $>800$ ha are left $[48,49]$ underlines the importance of conservation efforts for western dry deciduous forests.

\section{CONCLUSIONS}

This study contributes to our knowledge about the genetic structure of solitary mammals and primates in particular. It was conducted on a broader geographical scale than a previous study and revealed a matrilineal structure on this scale. Furthermore, we showed that gene flow in Mirza coquereli is male-biased and that this can lead to high genetic variation for codominant markers, such as microsatellites within a population, but also to discrepancies between codominant and haploid marker analyses. Hence, genetic studies investigating the evolutionary history of a species should take into account more than one genetic marker to prevent marker-biased results [50]. Finally, we showed that a solitary species is able to compensate drastic demographic fluctuations, if continuous habitats allow migration between subpopulations and, thus, to maintain overall genetic diversity.

\section{ACKNOWLEDGEMENTS}

We thank Profs. Olga Ramilijaona and Daniel Rakotondravony of the Département Biologie Animale de l'Université d'Antananarivo, the members of the Commission Tripartite and the C.A.F.F. of the Direction des Eaux et Forêts, and the CFPF Morondava for their authorization and support of this study. Thanks also to Melanie Dammhahn, Claudia Fichtel and all assistants of the l'équipe DPZ for their help in the field and to Linn Groeneveld, Nico Westphal and especially Christina Oberdieck for their support in the lab. We are also very grateful for financial support by the Margot Marsh Biodiversity Foundation and the Gesellschaft für Primatologie (GFP).

\section{REFERENCES}

[1] Jones ME, Paetkau D, Geffen E, Moritz C. Genetic diversity and population structure of Tasmanian devils, the largest marsupial carnivore. Mol Ecol 2004; 13: 2197-209.

[2] Keller LF, Jeffery KJ, Arcese P, Beaumont MA, Hochachka WM, Smith JN, et al. Immigration and the ephemerality of a natural population bottleneck: evidence from molecular markers. Proc $\mathrm{R}$ Soc Lond B 2001; 268:1387-94.

[3] Lande R. Genetics and demography in biological conservation. Science 1988; 241: 1455-60.

[4] Nei M, Maruyama T, Chakraborty R. The bottleneck effect and genetic variability in populations. Evolution 1975; 29: 1-10.

[5] Nystrom V, Angerbjorn A, Dalen L. Genetic consequences of a demographic bottleneck in the Scandinavian arctic fox. Oikos 2006; 114: 84-94.

[6] Wright S. Evolution and the genetics of populations. 2 vol. The theory of gene frequencies. Chicago: University of Chicago Press 1969.

[7] Frankham R, Ballou J, Briscoe D. Introduction to conservation genetics. Cambridge: Cambridge University Press 2002.

[8] Vila C, Sundqvist AK, Flagstad O, Seddon J, Bjornerfeldt S, Kojola I, et al. Rescue of a severely bottlenecked wolf (Canis lupus) population by a single immigrant. Proc R Soc Lond B 2003; 270: 91-7.

[9] Fredsted T, Pertoldi C, Schierup MH, Kappeler PM. Microsatellite analyses reveal fine-scale genetic structure in grey mouse lemurs (Microcebus murinus). Mol Ecol 2005; 14: 2363-72.

[10] Sugg D, Chesser R, Dobson F, Hoogland J. Population genetics meets behavioral ecology. Trends Ecol Evol 1996; 11: 338-342

[11] Glenn T, Stephan W, Braun M. Effects of a population bottleneck on whooping crane mitochondrial DNA variation. Conserv Biol 1999; 13: 1097-107.

[12] Lehman SM. Conservation biology of malagasy strepsirhines: A phylogenetic approach. Am J Phys Anthropol 2006; 130: 238-53.

[13] Hladik A, Charles-Dominique P, Petter J-J. Feeding strategies of five nocturnal prosimians in the dry forest of the west coas of Madagascar. In: Charle-Dominique P, Cooper H, Hladik A, Hladik C, Pages E, Pariente G, et al., eds. Nocturnal malagasy primates: behaviour, ecology and physiology. New York: Academic Press 1980: 41-73. 
[14] Kappeler PM, Wimmer B, Zinner D, Tautz D. The hidden matrilineal structure of a solitary lemur: implications for primate social evolution. Proc R Soc Lond B 2002; 269: 1755-63.

[15] Pages E. Ethoecology of Microcebus coquereli during the dry season. In: Charle-Dominique P, Cooper H, Hladik A, Hladik C, Pages E, Pariente G, et al., eds. Nocturnal malagasy primates: behaviour, ecology and physiology. New York: Academic Press 1980: 97-116.

[16] Kappeler PM. Intrasexual selection in Mirza coquereli: Evidence for scramble competition polygyny in a solitary primate. Behav Ecol Sociobiol 1997; 41: 115-27.

[17] Kappeler PM. Mirza coquereli, Coquerel's Dwarf Lemur. In: Goodman S, Benstead J, eds. The Natural History of Madagascar. Chicago: University Press 2003:1316-20.

[18] Stanger K, Coffmann B, Izard M. Reproduction in Coquerel's Dwarf Lemur (Mirza coquereli). Am J Primatol 1995; 36: 223-37.

[19] Yoder A, Irwin J, Goodman S, Rakotoarisoa S. Genetic tests of the taxonomic status of the ring-tailed lemur (Lemur catta) from the high mountain zone of the Andringitra Massif, Madagascar. J Zool 2000; 252: 1-9.

[20] Posada D. Collapse Version 1.2. 2004. Available from http:// darwin.uvigo.es/software/collapse.html

[21] Hapke A, Eberle M, Zischler H. Isolation of new microsatellite markers and application in four species of mouse lemurs (Microcebus sp.). Mol Ecol Notes 2003; 3: 205-8.

[22] Hapke A, Schulke O, Zischler H. Microsatellite markers for paternity testing in fork-marked lemurs (Phaner furcifer). Mol Ecol Notes 2003; 3: 438-40.

[23] Radespiel U, Funk SM, Zimmermann E, Bruford MW. Isolation and characterization of microsatellite loci in the grey mouse lemur (Microcebus murinus) and their amplification in the family Cheirogaleidae. Mol Ecol Notes 2001; 1: 16-8.

[24] Rozas J, Sanchez-DelBarrio JC, Messeguer X, Rozas R. DnaSP, DNA polymorphism analyses by the coalescent and other methods. Bioinformatics 2003; 1219: 2496-7.

[25] Peakall R, Smouse P. GENALEX 6: genetic analysis in Excel. Population genetic software for teaching and research. Mol Ecol 2006; 6: 288-95.

[26] Goudet J. FSTAT version 1.2 (version 2.9.3.2): a computer program to calculate F-statistics. J Hered 1995; 86: 485-6.

[27] van Oosterhout C, Hutchinson W, Wills D, Shipley P. MICROCHECKER: software for identifying and correcting genotyping errors in microsatellite data. Mol Ecol Notes 2004; 4: 535-8.

[28] Tajima F. The effect of change in population size on DNA polymorphism. Genetics 1989; 123: 597-601.

[29] Fu YX, Li WH. Statistical tests of neutrality of mutations. Genetics 1993; 133: 693-709.

[30] Rogers AR, Harpending H. Population growth makes waves in the distribution of pairwise genetic differences. Mol Biol Evol 1992; 9: 552-69.

[31] Piry S, Luikart G, Cornuet J-M. BOTTLENECK: A computer program for detecting recent reductions in the effective population size using allele frequency data. J Hered 1999; 90: 502-3.

[32] Luikart G, Allendorf F, Cornuet J-M, Sherwin W. Distortion of allele frequency distributions provides a test for recent population bottlenecks. J Hered 1998; 89: 238-47.

[33] Peakall R, Smouse PE, Huff D. Evolutionary implications of allozyme and RAPD variation in diploid populations of dioecious buffalograss, Buchloe dactyloides. Mol Ecol 1995; 4: 135-47.
[34] Smouse PE, Peakall R. Spatial autocorrelation analysis of individual multiallele and multilocus genetic structure. Heredity 1999; 82: 561-73.

[35] Dammhahn M, Kappeler PM. Social system of Microcebus berthae, the world's smallest primate. Int J Primatol 2004; 26: 407-35.

[36] Fredsted T, Pertoldi C, Olesen J, Eberle M, Kappeler PM. Microgeographic heterogeneity in spatial distribution and mtDNA variability of gry mouse lemurs (Microcebus murinus, Primates: Cheirogaleidae). Behav Ecol Sociobiol 2004; 56: 393-403.

[37] Fredsted T, Schierup M, Groeneveld L, Kappeler P. Genetic structure, lack of sex-biased dispersal and behavioral flexibility in the pair-living fat-tailed dwarf lemur, Cheirogaleus medius. Behav Ecol Sociobiol 2007; 61: 943-54.

[38] Escorza-Trevino S, Dizon AE. Phylogeography, intraspecific structure and sex-biased dispersal of Dall's porpoise, Phocoenoides dalli, revealed by mitochondrial and microsatellite DNA analyses. Mol Ecol 2000; 9: 1049-60.

[39] Nyakaana S, Arctander P. Population genetic structure of the African elephant in Uganda based on variation at mitochondrial and nuclear loci: evidence for male-biased gene flow. Mol Ecol 1999; 8: 1105-15.

[40] Muwanika V, Nyakaana S, Siegismund H, Arctander P. Population genetic structure of the common warthog (Phacochoerus africanus) in Uganda: evidence for a strong philopatry among warthogs and social structure breakdown in a disturbed population. Afr J Ecol 2006; 45: 22-30.

[41] Sanetra M, Crozier R. Patterns of population subdivision and gene flow in the ant Nothomyrmecia macrops reflected in microsatellite and mitochondrial markers. Mol Ecol 2003; 12: 2281-95.

[42] Haavie J, Saetre G-P, Moum T. Discrepancies in population differentiation at microsatellites, mitochondrial DNA and plumage colour in the pied flycatcher- inferring evolutionary processes. Mol Ecol 2000: 1137-48.

[43] Handley LJ, Perrin N. Advances in our understanding of mammalian sex-biased dispersal. Mol Ecol 2007; 16: 1559-78.

[44] Yao Y, Nie L, Harpending H, Fu Y, Yuan Z, Zhang Y. Genetic Relationship of Chinese Ethnic Populations Revealed by mtDNA Sequence Diversity. Am J Phys Anthropol 2002; 118: 68-76.

[45] Radespiel U, Ehresmann P, Zimmermann E. Species-specific usage of sleeping sites in two sympatric mouse lemur species (Microcebus murinus and M. ravelobensis) in northwestern Madagascar. Am J Primatol 2003; 59(4): 139-51.

[46] Wimmer B, Tautz D, Kappeler PM. The genetic population structure of the grey mouse lemur (Microcebus murinus), a basal primate from Madagascar. Behav Ecol Sociobiol 2002; 52: 166-75.

[47] Whitmore T. Madagascar deforestation rate during 1980. In: Lourenço W, Goodman S, eds. Biogeography of Madagascar. Paris: Mémoires de la Société de Biogéographie 2000: 126.

[48] Ganzhorn J, Lowry II P, Schatz G, Sommer S. The biodiversity of Madagascar: one of the world's hottest hotspots on its way out. Oryx 2001; 35: 346-8.

[49] Smith A. Deforestation, fragmentation, and reserve design in western Madgascar. In: Lawrence W, Bierregaard O, eds. Tropical Forest Remnants, Ecology, management and Conservation of Fragmented Communities. Chicago: University of Chicago Press 1997: 415-41.

[50] Melnick D, Hoelzer G. Differences in Male and Female Macaque Dispersal Lead to Contrasting Distributions of Nuclear and Mitochondrial DNA Variation. Int J Primatol 1992; 13: 379-93.

Received: February 27, $2008 \quad$ Revised: May 06, $2008 \quad$ Accepted: June 16, 2008

(C) Markolf; Licensee Bentham Open.

This is an open access article distributed under the terms of the Creative Commons Attribution License (http://creativecommons.org/licenses/by/2.5/), which permits unrestrictive use, distribution, and reproduction in any medium, provided the original work is properly cited. 